\title{
ON THE HASSE PRINCIPLE FOR SHIMURA CURVES
}

\author{
PETE L. CLARK
}

\begin{abstract}
Let $C$ be an algebraic curve defined over a number field $K$, of positive genus and without $K$-rational points. We conjecture that there exists some extension field $L$ over which $C$ violates the Hasse principle, i.e., has points everywhere locally but not globally. We show that our conjecture holds for all but finitely many Shimura curves of the form $X_{0}^{D}(N) / \mathbb{Q}$ or $X_{1}^{D}(N) / \mathbb{Q}$, where $D>1$ and $N$ are coprime squarefree positive integers. The proof uses a variation on a theorem of Frey, a gonality bound of Abramovich, and an analysis of local points of small degree.
\end{abstract}

\section{INTRODUCTION}

Fix $D>1$ a squarefree positive integer, and $N \geq 1$ a squarefree positive integer which is coprime to $D$. Then there exist Shimura curves $X_{0}^{D}(N) / \mathbb{Q}$ (resp. $\left.X_{1}^{D}(N) / \mathbb{Q}\right)$, which are moduli spaces for abelian surfaces endowed with endomorphisms by a maximal order in the indefinite rational quaternion algebra $B_{D}$ of discriminant $D$ and $\Gamma_{0}(N)$ (resp. $\Gamma_{1}(N)$ ) level structure. There are natural ("forgetful") modular maps $X_{1}^{D}(N) \rightarrow X_{0}^{D}(N) \rightarrow X^{D}=X_{0}^{D}(1)$.

Understanding the points on these curves rational over various number fields $K$ and their completions is an important problem, analogous to the corresponding problem for the classical modular curves $X_{1}(N), X_{0}(N)$ but even more interesting in at least one respect. Namely, although the classical modular curves have $\mathbb{Q}$-rational cusps, we have $X^{D}(\mathbb{R})=\emptyset$. This raises the possibility that certain Shimura curves, when considered over suitable non-real number fields $K$, may violate the Hasse principle, i.e., may have points rational over every completion of $K$ but not over $K$ itself. And indeed, Jordan showed that the curve $X_{/ \mathbb{Q}(\sqrt{-23})}^{39}$ violates the Hasse principle [10] (see also [16). Later, Skorobogatov and Yafaev provided explicit conditions on $D, N$ and $K=\mathbb{Q}(\sqrt{m})$ sufficient for $X_{0}^{D}(N) / K$ to violate the Hasse principle [17. Although it is plausible that their conditions can be met for infinitely many choices of $(D, N, K)$, these conditions (like Jordan's) include hypotheses about the class group of $K$, so proving that they hold infinitely often seems very difficult.

Using different methods, we shall show that almost all Shimura curves of squarefree level violate the Hasse principle. More precisely:

Theorem 1. If $D>546$, there is an integer $m$ such that $X_{/ \mathbb{Q}(\sqrt{m})}^{D}$ violates the Hasse principle.

Theorem 2. There exists a constant $C$ such that if $D \cdot N>C$, then there exist number fields $K=K(D, N)$ and $L=L(D, N)$ such that $X_{0}^{D}(N) / K$ and $X_{1}^{D}(N) / L$ violate the Hasse principle. 
Theorem 3. Maintain the notation of the previous theorem, and assume $D \cdot N>C$. a) We may choose $K$ such that $[K: \mathbb{Q}] \mid 4$.

b) Let $\left\{N_{i}\right\}$ be a sequence of squarefree positive integers tending to infinity, and for each $i$, choose any squarefree positive integer $D_{i}>1$ which is prime to $N_{i}$ and such that $D_{i} \cdot N_{i}>C$. For all $i$, choose any number field $L_{i}$ such that $X_{1}^{D_{i}}\left(N_{i}\right)_{/ L_{i}}$ violates the Hasse Principle. Then $\lim _{i \rightarrow \infty}\left[L_{i}: \mathbb{Q}\right]=\infty$.

Remark: Using the same methods, one can show that there exists a positive integer $G$ such that if $F$ is a totally real field and $X_{/ F}$ is a semistable Shimura curve of squarefree level of genus greater than $G$, then there exists an extension $K / F$ of degree dividing 4 such that $X_{/ K}$ violates the Hasse principle. (Presumably the case of $\Gamma_{1}(N)$-level structure generalizes as well; I have not checked the details.)

We choose to view these results as special cases of a more general conjecture on algebraic curves defined over number fields. Let $V$ be a nonsingular, geometrically irreducible variety defined over a number field $K$. If there exists a number field $L / K$ such that $V_{/ L}$ has points rational over every completion of $L$ but no $L$-rational points, we say that $V_{/ K}$ is a potential Hasse principle violation (or for brevity, " $V_{/ K}$ is PHPV.")

Obviously $V_{/ K}$ can only be PHPV if it has no $K$-rational points. Moreover, restricting to the class of curves, the case of genus zero must be excluded, by HasseMinkowksi. No further restrictions spring to mind, and we conjecture that there are none:

Conjecture 4. Let $C_{/ K}$ be a curve defined over a number field $K$. Assume that:

(i) $C$ has no $K$-rational points.

(ii) $C$ has positive genus.

Then $C_{/ K}$ is a potential Hasse principle violation.

A proof of this conjecture in the general case may not be within current reach. However, using work of Faltings and Frey we will derive a criterion for $C_{/ K}$ to be PHPV (Theorem 6). We use this criterion, together with an analysis of local points on Shimura curves and a result of Abramovich, to prove Theorems 1-3.

Finally, a warning: our method does not give an effective procedure for finding the fields $K$ and $L$. This is to be contrasted with the work of [10] and [17.

Remark and acknowledgment: I showed that there exist infinitely many Shimura curves $X_{0}^{D}(N)$ violating the Hasse principle over suitable quadratic fields in my 2003 Harvard thesis 4, Main Theorem 5]. Recently I became encouraged by the interest in this unpublished work shown by V. Rotger, A. Skorobogatov and A. Yafaev [15] and decided at last to write up the result. Several improvements were found along the way, and the present results are stronger (and in some ways simpler) than what appeared in [4. I would like to take this opportunity - better late than never - to gratefully acknowledge the support of Harvard University and of my thesis advisor, Barry Mazur. 


\section{Criteria for a Curve to Be PHPV}

Let $X_{/ K}$ be a variety over a field $K$. Define the $m$-invariant $m(X)=m\left(X_{/ K}\right)$ to be the minimum degree of a finite field extension $L / K$ such that $X(L) \neq \emptyset$.

If $K$ is a number field and $v$ is a place of $K$, we put $m_{v}(X):=m\left(X_{/ K_{v}}\right)$ and $m_{\mathrm{loc}}(X)=\operatorname{lcm}_{v} m_{v}(X)$. Note that applying Bertini's theorem and the Weil bound for curves over finite fields, one gets the (well known) fact that $m_{v}(X)=1$ for all but finitely many $v$, so $m_{\text {loc }}$ is well-defined.

The $K$-gonality of a curve $C_{/ K}$, denoted $d=d_{K}(C)$, is the least positive integer $n$ for which there exists a degree $n$ morphism $\varphi_{/ K}: C \rightarrow \mathbb{P}^{1}$. Clearly $d_{K}(C)=1 \Longleftrightarrow C \cong_{K} \mathbb{P}^{1}$. We say $C_{/ K}$ is hyperelliptic if $d_{K}(C) \leq 2$. (Beware that this is not quite the standard definition: under our definition all curves of genus zero and some curves of genus one are hyperelliptic, and a curve of higher genus may be hyperelliptic over $\bar{K}$ but not over $K$.)

For any curve $C_{/ K}$ we have $m(C) \leq d_{K}(C)$. Indeed, the preimages of $\mathbb{P}^{1}(K)$ under a degree $d=d_{K}(C)$ morphism $\varphi: C \rightarrow \mathbb{P}^{1}$ yield infinitely many points $P$ on $C$ of degree at most $d$. Lying very much deeper is the following result, which is a sort of converse.

Theorem 5. Let $C_{/ K}$ be a curve over a number field, and, for $n \in \mathbb{Z}^{+}$, let $\mathcal{S}_{n}(C)$ be the set of points $P \in C(\bar{K})$ of degree dividing $n$. If $\mathcal{S}_{n}(C)$ is infinite, then $d_{K}(C) \leq 2 n$.

Proof: This is a small variation on a result of Frey [7 Prop. 2] (which itself uses Faltings' spectacular theorem on rational points on subvarieties of abelian varieties). In the original version, instead of $\mathcal{S}_{n}(C)$ there appears the set $C^{(d)}(K)$ of points of degree less than or equal to $d$. However, there also appears the extra hypothesis that there exists $P_{0} \in C(K)$, which must be removed for our applications. The existence of $P_{0}$ is used to define a map from the $d$-fold symmetric product $C^{(d)}$ to the Jacobian $\operatorname{Jac}(C)$, namely

$$
\Phi: P_{1}+\ldots+P_{d} \mapsto\left[P_{1}+\ldots+P_{d}-d P_{0}\right] .
$$

However, if $\mathcal{S}_{n}(C)$ is infinite, it is certainly nonempty, so that for some $m \mid n$ there exists an effective $K$-rational divisor $D_{m}$ of degree $m$, hence indeed an effective $K$-rational divisor of degree $n$, namely $D_{n}=\left(\frac{n}{m}\right) D_{m}$. Then one can define the map

$$
\Phi_{D}: P_{1}+\ldots+P_{d} \mapsto\left[P_{1}+\ldots+P_{d}-D_{n}\right],
$$

and Frey's argument goes through verbatim with $\Phi_{D}$ in place of $\Phi$.

Theorem 6. Let $C_{/ K}$ be an algebraic curve defined over a number field. Suppose: a) $C(K)=\emptyset$.

b) $d_{K}(C)>2 m>2$ for some multiple $m$ of $m_{\mathrm{loc}}(C)$.

Then there exist infinitely many extensions $L / K$ with $[L: K]=m$ such that $C_{/ L}$ is a counterexample to the Hasse principle.

Proof: By Theorem $\left[\mathcal{S}_{m}(C)\right.$ is a finite set. It follows that the field $M$ defined as the compositum of $K(P)$ as $P$ ranges through elements of $\mathcal{S}_{m}(C)$, is a number field. Let $L / K$ be a number field which is linearly disjoint from $M / K$ and such 
that $[L: K]=m$. Since $C(K)=\emptyset$, it follows that $C(L)=\emptyset$.

Let $S=\left\{v_{1}, \ldots, v_{r}\right\}$ be the places of $K$ for which $m_{v_{i}}(C)>1$. By definition of $m_{\mathrm{loc}}(C)=\operatorname{lcm}_{v} m_{v}(C)$, for each finite place $v_{i} \in S$, there exists a field extension $L_{i} / K_{v_{i}}$ of degree ( $m_{\mathrm{loc}}$, and a fortiori of degree) $m$ such that $C\left(L_{i}\right)$ is nonempty. At each Archimedean place $v_{i} \in S$ (if any), we take $L_{i}$ to be the $\mathbb{R}$-algebra $\mathbb{C}^{\frac{m}{2}}$. Let $v_{0}$ be any finite place not in $\Sigma$ and unramified in $M$, and let $L_{0} / K_{v_{0}}$ be a totally ramified extension of degree $m$. For $0 \leq i \leq r$ let $f_{i} \in K_{v_{i}}[x]$ be a defining polynomial for $L_{i} / K_{v_{i}}$. By weak approximation, for any $\epsilon>0$, there exists a degree $m$ polynomial $f \in K[x]$ such that, for all $i$, each coefficient of $f-f_{i}$ has $v_{i}$-adic norm at most $\epsilon$, so by Krasner's Lemma, for sufficiently small $\epsilon, L=K[x] /(f)$ defines a degree $m$ field extension with $L \otimes_{K} K_{v_{i}} \cong L_{i}$ for $0 \leq i \leq r$. By construction $m_{\text {loc }}\left(C_{/ L}\right)=1$; moreover, $L / K$ is disjoint from $M / K$, so $C(L)=\emptyset$. By varying the choice of $v_{0}$ we clearly get infinitely many distinct fields $L$.

For $1 \leq n \leq 3$ there is a complete classification of algebraic curves which have infinitely many points of degree at most $n$ (Faltings for $n=1$, [8 for $n=2$, 2 for $n=3$ ). The quadratic case leads to the following "supplement" to Theorem [6]

Theorem 7. Suppose $C_{/ K}$ is a curve over a number field with $m_{\mathrm{loc}}(C)=2$, $d_{K}(C)>2$, and $C$ does not admit a degree two morphism $\varphi: C \rightarrow E$, where $E_{/ K}$ is an elliptic curve of positive rank. Then there exist infinitely many quadratic field extensions $L / K$ such that $C_{/ L}$ is a counterexample to the Hasse principle.

Proof: By the main result of $\left[\underline{8}\right.$, the hypotheses imply that $\mathcal{S}_{2}(C)$ is finite, and the rest of the proof is the same as that of Theorem 6

\section{Local POINTS on Shimura CURVES}

We recall our notation: $D$ is the discriminant of a nonsplit indefinite rational quaternion algebra $B_{D}$, and is thus a nontrivial product of an even number of primes; $N$ is a squarefree positive integer prime to $D$.

The goal of this section is to prove the following result.

Theorem 8. a) For all $D, m_{\mathrm{loc}}\left(X^{D}\right)=2$.

b) For all $D$ and all $N, m_{\mathrm{loc}}\left(X_{0}^{D}(N)\right)$ is either 2 or 4 .

Remark: Of course, for part b) one would like to know which of the two alternatives obtains. It is possible to give a precise answer for this; more exactly, there are several nice sufficient conditions for $m_{\mathrm{loc}}\left(X_{0}^{D}(N)\right)=2$ - this holds, e.g., for fixed $D$ and all sufficiently large prime numbers $N$ - and if none of these sufficient conditions hold there is a straightforward finite computation (coming from the Eichler-Selberg trace formula) that will determine the answer for any given pair $(D, N)$. We hope to return to this and other matters pertaining to quadratic points on Shimura curves in a future work.

3.1. Integral structure. By work of Morita and Drinfeld there exists an integral canonical model for $X_{0}^{D}(N)$. In more words, there is a $\mathbb{Z}$-scheme, projective, flat, and of relative dimension one, and which is a coarse moduli space for Drinfeld's extension of the moduli problem defining $X_{0}^{D}(N)_{\mathbb{Q}}$ to the category of schemes over $\mathbb{Z}$. The generic fiber is canonically isomorphic to $X_{0}^{D}(N)$, so we may denote this scheme by $X_{0}^{D}(N)_{\mathbb{Z}}$. In particular, for every prime number $p$ we have a special fiber 
$X_{0}^{D}(N)_{\mathbb{F}_{p}}$, whose structure is highly relevant in the computation of $m_{p}\left(X_{0}^{D}(N)\right)$. Depending upon $p$, the special fiber exhibits three different kinds of behavior.

3.2. Case of good reduction. If $(p, D N)=1, X_{0}^{D}(N) / \mathbb{Z}_{p}$ is smooth.

As above, the special fiber $X_{0}^{D}(N)_{/ \mathbb{F}_{p}}$ is again a moduli space of abelian surfaces $A_{/ k}$ (where $k$ is a field of characteristic $p$ ) endowed with a quaternionic structure, i.e., an injection $\mathcal{O} \hookrightarrow$ End $A$. However, in contrast to characteristic zero, where the generic QM surface is geometrically simple, all QM surfaces in characteristic $p$ are isogenous to $E \times E$, where $E_{/ k}$ is an elliptic curve [12]. Thus the full endomorphism ring of $A$ depends on $\operatorname{End}(E)$ : it is most often an order in an imaginary quadratic field - in which case we say $A$ (and its corresponding point on the moduli space) is ordinary - but there is a finite non-empty set of points for which the endomorphism ring of $E$ is an order in a definite rational quaternion algebra (of discriminant $p$ ). The union of such points determines the supersingular locus on $X_{/ \mathbb{F}_{p}}^{D}$, and the supersingular locus on $X_{0}^{D}(N)_{/ \mathbb{F}_{p}}$ is its complete preimage under the modular "forgetful" map $X_{0}^{D}(N) \rightarrow X^{D}$.

Proposition 9. There exists at least supersingular point on $X_{0}^{D}(N)_{\mathbb{F}_{p}}$. Moreover, every supersingular point is defined over $\mathbb{F}_{p^{2}}$.

Proof: This is well known; see e.g. [13].

Corollary 10. For p prime to $N D, m_{p}\left(X_{0}^{D}(N)\right) \leq 2$.

Proof: By Proposition $9 m\left(X_{0}^{D}(N)_{\mathbb{F}_{p}}\right) \leq 2$. Since $X_{0}^{D}(N)$ is smooth over $\mathbb{Z}_{p}$, Hensel's Lemma implies that the $m$-invariant of the generic fiber is at most the $m$-invariant of the special fiber, and the result follows.

3.3. Case of Cerednik-Drinfeld reduction. For a positive integer $a$, we shall write $\mathbb{Q}_{p^{a}}$ for the unramified extension of $\mathbb{Q}_{p}$ of degree $a$ and $\mathbb{Z}_{p^{a}}$ for its valuation ring.

If $p \mid D$, then there exists a Mumford curve $C(D, N)_{/ \mathbb{Z}_{p}}$ whose basechange to $\mathbb{Z}_{p^{2}}$ is isomorphic to $X_{0}^{D}(N) / \mathbb{Z}_{p^{2}}$.

Recall that a Mumford curve $C_{/ \mathbb{Q}_{p}}$ is an algebraic curve which can be uniformized by the $p$-adic upper half-plane. Equivalently, the special fiber of the minimal regular model $\mathcal{C}_{\mathbb{Z}_{p}}$ is a semistable curve which $\mathbb{F}_{p}$-split and degenerate: every irreducible component is isomorphic to $\mathbb{P}^{1}$, with transverse intersections at $\mathbb{F}_{p}$-rational points. The special fiber is thus completely determined by its dual graph, a finite graph in which the vertices correspond to the irreducible components $C_{i}$ and the edges correspond to intersection points of $C_{i}$ and $C_{j}$. Moreover, the degree of each vertex is at most $p+1$.

Proposition 11. If $p \mid D, m_{p}\left(X_{0}^{D}(N)\right) \leq 2$.

Proof: By Hensel's Lemma, it is enough to find a smooth $\mathbb{F}_{p^{2}}$-rational point on $X_{0}^{D}(N) \cong_{\mathbb{Z}_{p^{2}}} C(D, N)$. But any irreducible component $C_{i}$ on $C(D, N)_{\mathbb{F}_{p}}$ has at most $p+1=\# \mathbb{P}^{1}\left(\mathbb{F}_{p}\right)$ singular points, hence at least $p^{2}+1-(p+1)>0$ smooth

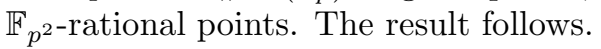




\subsection{Case of Deligne-Rapoport reduction. Suppose $p \mid N$.}

Theorem 12. The arithmetic surface $X_{0}^{D}(N)_{/ \mathbb{Z}_{p}}$ has the following structure:

a) The special fiber $X_{0}^{D}(N)_{\mathbb{F}_{p}}$ has two irreducible components, each isomorphic to the (smooth) curve $X_{0}^{D}\left(\frac{N}{p}\right)_{\mathbb{F}_{p}}$.

b) The two irreducible components intersect transversely at the supersingular points, a corresponding point on the first copy of $X_{0}^{D}\left(\frac{N}{p}\right)_{\mathbb{F}_{p}}$ being glued to its image under the Frobenius map.

c) At each supersingular point $z \in X_{0}^{D}(N)_{/ \mathbb{F}_{p^{2}}}$, the complete local ring is isomorphic to $\mathbb{Z}_{p^{2}}[[X, Y]] /\left(X Y-p^{a_{z}}\right)$ for some positive integer $a_{z}$.

Proof: The analogous statement for $D=1$ (i.e., classical modular curves) is due to Deligne and Rapoport [6]. In that same work it was pointed out that their result would continue to hold in the quaternionic context. Apparently the first careful treatment of the quaternionic case is due to Buzzard [3], who worked however under the assumption of some additional rigidifying level structure. The case of $X_{0}^{D}(N)$ (which is not a fine moduli scheme) was worked out (independently) in the theses of the author (unpublished) and of David Helm [9, Appendix].

Proposition 13. For any prime $p \mid N, m_{p}\left(X_{0}^{D}(N)\right) \leq 4$.

Proof: Consider $X_{0}^{D}(N)_{\mathbb{F}_{p^{2}}}$. Let $z$ be any point on this curve coming from a supersingular point of $X_{0}^{D}\left(\frac{N}{p}\right)\left(\mathbb{F}_{p^{2}}\right)$. The local ring at $z$ is analytically isomorphic to $\mathbb{Z}_{p^{2}}[[x, y]] /\left(x y-p^{a}\right)$ for some integer $a \geq 1$.

Suppose first that $a>1$. Then in order to get the minimal regular model, one must blow up the point $z(a-1)$ times, getting a chain of $a-1$ rational curves defined over $\mathbb{F}_{p^{2}}$. Each of these curves has $p^{2}+1-2$ smooth $\mathbb{F}_{p^{2}}$-rational points, which lift to give points on $X_{0}^{D}(N)$ rational over $\mathbb{Q}_{p^{2}}$, the unramified quadratic extension.

Now assume that we have $a=1$. Let $K=\mathbb{Q}_{p^{2}}(\sqrt{p})$, with valuation ring $R$ and uniformizing element $\pi=\sqrt{p}$. Then the completed local ring at $z$ of the integral model $X_{0}^{D}(N)_{/ R}$ is isomorphic to $R[[x, y]] /\left(x y-\pi^{2}\right)$. We see that this local ring is no longer regular and must be blown up once to give a rational curve on the regular model. Thus, as in the previous case, we get a $K$-rational point on $X_{0}^{D}(N)$, completing the proof.

Proof of Theorem 8 From Propositions 10 11] and 13] we get $m_{\mathrm{loc}}\left(X_{0}^{D}(N)\right) \mid 4$. Recalling that $X_{0}^{D}(N)(\mathbb{R})=\emptyset$, the result follows.

\section{LOWER BOUNDS ON THE GONALITY AND GENUS}

4.1. A result of Abramovich. Let $\mathcal{O}^{1}$ be the group of norm 1 units in a maximal $\mathbb{Z}$-order $\mathcal{O}$ of $B_{D}$. By restricting any embedding $B \hookrightarrow B \otimes_{\mathbb{Q}} \mathbb{R} \cong M_{2}(\mathbb{R})$ to $\mathcal{O}^{1}$, one gets a realization of $\mathcal{O}^{1}$ as a discrete cocompact subgroup of $S L_{2}(\mathbb{R})$, welldetermined up to conjugacy. Then $\mathcal{O}^{1} \backslash \mathcal{H}$ is a compact Riemann surface isomorphic to $X^{D}(\mathbb{C})$. For any finite index subgroup $\Gamma \subset \mathcal{O}^{1}$, we get a covering $X(\Gamma) \rightarrow X^{D}$.

Theorem 14. (Abramovich)

$$
\frac{21}{200}\left(g\left(X^{D}(\Gamma)\right)-1\right) \leq d_{\mathbb{C}}\left(X^{D}(\Gamma)\right) .
$$


4.2. Genus estimates. In this section, all asymptotics are as $\min (D, N) \rightarrow \infty$.

For coprime squarefree positive integers $A$ and $N$, define

$$
\begin{aligned}
& e_{2}(D, N)=\prod_{p \mid D}(1-(-4 / p)) \prod_{q \mid N}(1+(-4 / q)), \\
& e_{3}(D, N)=\prod_{p \mid D}(1-(-3 / p)) \prod_{q \mid N}(1+(-3 / q)) .
\end{aligned}
$$

Let $\varphi$ be Euler's function, i.e., the multiplicative function such that $\varphi\left(p^{k}\right)=$ $p^{k}-p^{k-1}$. Let $\psi$ be the multiplicative function such that $\psi\left(p^{k}\right)=p^{k}+p^{k-1}$.

Then we have [13 p. 301]:

$$
g\left(X_{0}^{D}(N)\right)=1+\frac{1}{12} \varphi(D) \psi(N)-\frac{e_{2}(D, N)}{4}-\frac{e_{3}(D, N)}{3} \sim \frac{\varphi(D) \psi(N)}{12} .
$$

In the case of $X_{1}^{D}(N)$ we will content ourselves with a lower bound for the genus. Indeed, we will work instead with the Shimura covering $X_{2}^{D}(N)$, which is by definition the largest intermediate covering

$$
X_{1}^{D}(N) \rightarrow Y \rightarrow X_{0}^{D}(N)
$$

such that $Y \rightarrow X_{0}^{D}(N)$ is unramified abelian, say with Galois group $\Sigma(D, N){ }^{1}$ From 11, Corollary 1], we get

$$
\# \Sigma(D, N) \geq \frac{\varphi(N)}{2 \cdot \prod_{q \mid N} 6} .
$$

Because $X_{2}^{D}(N) \rightarrow X_{0}^{D}(N)$ is unramified, we have

$$
g\left(X_{2}^{D}(N)\right)-1=\# \Sigma(D, N)\left(g\left(X_{0}^{D}(N)\right)-1\right),
$$

and combining equations (2) through (4), we get

$$
g\left(X_{1}^{D}(N)\right)-1 \geq g\left(X_{2}^{D}(N)\right) \gg \varphi(N) \cdot \frac{\varphi(D)}{24} \cdot \prod_{q \mid N} \frac{q+1}{6} .
$$

4.3. Proofs of the main theorems. It is now only a matter of combining the results of $\S 2, \S 3$ and $\S 4$.

Consider first the case of $D=1$. According to Theorem $8 m_{\text {loc }}\left(X^{D}\right)=2$. Moreover, by [14, $X^{D}$ has only finitely many quadratic points when $D>546$. Thus Theorem 7 applies to show that for such $D$, there exist infinitely many quadratic fields $K$ such that $X_{/ K}^{D}$ violates the Hasse principle.

Next consider the case of $X_{0}^{D}(N)$. By Theorem 8 we have $2\left|m_{\text {loc }}\left(X_{0}^{D}(N)\right)\right| 4$; in particular, $X_{0}^{D}(N)(\mathbb{Q})=\emptyset$. Applying Theorem 6 with $m=4$, we get that whenever the gonality $d_{\mathbb{Q}}\left(X_{0}^{D}(N)\right)>8$, there exists infinitely many quartic fields $K / \mathbb{Q}$ such that $X_{0}^{D}(N) / K$ violates the Hasse principle. But by (1) and $(2), d_{\mathbb{Q}}\left(X_{0}^{D}(N)\right) \geq$ $d_{\mathbb{C}}\left(X_{0}^{D}(N)\right) \gg \varphi(D) \psi(N)$. Since the latter quantity approaches infinity with $\min (D, N)$, the gonality condition holds for all but finitely many pairs $(D, N)$.

\footnotetext{
${ }^{1}$ We introduce the Shimura covering only to save a couple of lines of messy calculation with the Riemann-Hurwitz formula.
} 
To deduce the $X_{1}^{D}(N)$ case, we need the following (essentially trivial) bound on the local m-invariant of $X_{1}^{D}(N)$.

Lemma 15. Let $K$ be a number field and $f_{/ K}: X_{1} \rightarrow X_{2}$ be a degree $d$ Galois covering of curves. Then $m_{\mathrm{loc}}\left(X_{1}\right) \mid d \cdot m_{\mathrm{loc}}\left(X_{2}\right)$.

Proof: First suppose that $f: X_{2} \rightarrow X_{1}$ is a Galois covering of curves defined over any field $K$, and let $P \in X_{1}(L)$ with $[L: K]=m\left(X_{1}\right)$. Recall that the transitivity of the action of $\operatorname{Gal}\left(L\left(X_{2}\right) / L\left(X_{1}\right)\right)$ on the points $\left\{Q_{1}, \ldots, Q_{g}\right\}$ of $X_{2}$ lying over $P$ implies the relation ef $g=d$, where $e$ is the relative ramification index of $Q_{i}$ over $P$ and $f=\left[L\left(Q_{i}\right): L\right]$. Thus $\left[L\left(Q_{i}\right): K\right]=f m\left(X_{1}\right) \mid d m\left(X_{1}\right)$. The result follows easily by applying this observation at every place of $K$.

\section{Proposition 16.}

$$
m_{\mathrm{loc}}\left(X_{1}^{D}(N)\right) \mid 2 \varphi(N) .
$$

Proof: The natural map $X_{1}^{D}(N) \rightarrow X_{0}^{D}(N)$ is a Galois covering with group $(\mathbb{Z} / N \mathbb{Z})^{\times} /( \pm 1)$. Thus the result follows from Theorem 8 and Lemma 15

Now

$$
\begin{gathered}
d_{\mathbb{Q}}\left(X_{1}^{D}(N)\right) \geq d_{\mathbb{C}}\left(X_{1}^{D}(N)\right) \geq \frac{21}{200}\left(g\left(X_{1}^{D}(N)-1\right) \geq \frac{21}{200}\left(g\left(X_{2}^{D}(N)\right)-1\right)\right. \\
\gg \varphi(N) \frac{\varphi(D)}{24} \prod_{q \mid N} \frac{q+1}{6},
\end{gathered}
$$

so that with $m=2 \varphi(N)$, we have

$$
\frac{d_{\mathbb{Q}}\left(X_{1}^{D}(N)\right)}{m} \gg \varphi(D) \prod_{q \mid N} \frac{q+1}{6} .
$$

Again the right-hand side approaches infinity with $\min (D, N)$, so except for finitely many pairs $(D, N)$, we can find a field $L$ of degree $2 \varphi(N)$ such that $X_{1}^{D}(N)_{/ L}$ violates the Hasse principle.

Finally, Theorem 3b) is an immediate consequence of the following:

Theorem 17. For each prime number $p$ and positive integer $d \geq 1$, there exists a constant $N_{0}=N_{0}(p, d)$ with the following property: for any $p$-adic field $K / \mathbb{Q}_{p}$ with $\left[K: \mathbb{Q}_{p}\right] \leq d$ and any integer $N \geq N_{0}, X_{1}^{D}(N)(K)=\emptyset$.

Proof: This is [5] Theorem1].

Indeed, taking, e.g., $p=2$, we have that $m_{2}\left(X_{1}^{D}(N)\right) \rightarrow \infty$ with $N$, uniformly in $D$.

\section{Final Remarks}

The constant $C$ in Theorem 1 can certainly be made explicit. As the reader has probably noticed, it is easy to do so assuming a fixed number of prime divisors of $D \cdot N$, but slightly tedious in the general case.

More interesting than whittling down the set of excluded pairs $(D, N)$ to the optimal list which violate the inequality $d_{\mathbb{Q}}\left(X_{0}^{D}(N)\right)>2 m_{\mathrm{loc}}\left(X_{0}^{D}(N)\right)$ is to investigate 
the cases of Conjecture 3 among Shimura curves of low (but positive!) genus. For instance, suppose $X_{0}^{D}(N)$ has genus one. All such curves can be given in the form $y^{2}=P(x)$, where $P \in \mathbb{Q}[x]$ has degree 4 (and distinct roots). For example, $X^{14}$ is given by the equation $\left(x^{2}-13\right)^{2}+7^{3}+2 y^{2}=0$. It is not hard to see that $X_{/ \mathbb{Q}(\sqrt{m})}^{14}$ has points everywhere locally if and only if $m$ is negative and prime to 7 , so that the set of such $m$ has density $\frac{3}{7}$ (as a subset of the set of all squarefree integers). What can be said about the set of $m$ for which $X^{14}(\mathbb{Q}(\sqrt{m}))$ is nonempty? Or even about its density?

One needs to know relatively little about the Shimura curves $X_{0}^{D}(N)$ beyond their semistability to show that almost all of them satisfy Conjecture 4 Indeed, one can show the following

Theorem 18. Let $\left\{X_{n}\right\}_{n=1}^{\infty}$ be a sequence of curves over a number field $K$.

Suppose:

a) Each $X_{n}$ has everywhere semistable reduction.

b) $\lim _{n \rightarrow \infty} \frac{d_{K}\left(X_{n}\right)}{\log g\left(X_{n}\right)}=\infty$.

c) There exists a fixed positive integer $A$ such that for all places $v$ and all $n$, the Galois action on the irreducible components of the special fiber $\left(X_{n}\right)_{/ k_{v}}$ of the minimal model trivializes over an extension of degree $A$.

Then $X_{n}$ is PHPV for all sufficiently large $n$.

The hypotheses of theorem are satisfied (with $A=2$ ) for the family of all semistable Shimura curves of squarefree level over any given totally real field $F$. Note that condition b) looks very mild: surely a "general" family of curves, semistable or otherwise, should have gonality on the order of $g\left(X_{n}\right)$.

I will save the proof for a later time, at which point I hope it will be more clear whether condition c) can be weakened or removed.

\section{REFERENCES}

[1] D. Abramovich, A linear lower bound on the gonality of modular curves, Internat. Math. Res. Notices 1996, 1005-1011.

[2] D. Abramovich and J. Harris, Abelian varieties and curves in $W_{d}(C)$, Compositio Math. 78 (1991), 227-238.

[3] K. Buzzard, Integral models of certain Shimura curves, Duke Math Journal, Duke Math. J. 87 (1997), 591-612.

[4] P.L. Clark, Rational points on Atkin-Lehner quotients of Shimura curves, 2003 Harvard thesis.

[5] P.L. Clark and X. Xarles, Local bounds for torsion points on abelian varieties, submitted for publication.

[6] P. Deligne and M. Rapoport, Les schémas de modules de courbes elliptiques, in: A. Dold and B. Eckmann (eds.) Modular Functions of One Variable II, Lecture Notes in Math. 349, Springer-Verlag, New York, (1973), 143-316.

[7] G. Frey, Curves with infinitely many points of fixed degree, Israel J. Math. 85 (1994), 79-83.

[8] J. Harris and J. Silverman, Bielliptic curves and symmetric products, Proc. Amer. Math. Soc. 112 (1991), 347-356.

[9] D. Helm, On maps between modular Jacobians and Jacobians of Shimura curves, submitted for publication.

[10] B.W. Jordan, Points on Shimura curves rational over number fields, J. Reine Angew. Math. 371 (1986), 92-114.

[11] S. Ling, Shimura subgroups of Jacobians of Shimura curves, Proc. Amer. Math. Soc. 118 (1993), 385-390. 
[12] J. Milne, Points on Shimura varieties mod p. Proc. Symp. Pure Math. XXXIII (1979), Part $2,165-184$

[13] A. Ogg, Real points on Shimura curves, Progr. Math. 35 (1983), Vol. I, 277-307.

[14] V. Rotger, On the group of automorphisms of Shimura curves and applications, Compositio Math. 132 (2002), 229-241.

[15] V. Rotger, A. Skorobogatov and A. Yafaev, Failure of the Hasse principle for Atkin-Lehner quotients of Shimura curves over $\mathbb{Q}$, Moscow Math. J. 5 (2002).

[16] A. Skorobogatov, Shimura coverings of Shimura curves and the Manin obstruction, Math. Research Letters 12 (2005), 779-788.

[17] A. Skorobogatov and A. Yafaev, Descent on certain Shimura curves, Israel J. Math. 140 (2004), 319-332.

1126 Burnside Hall, Department of Mathematics and Statistics, McGill University, 805 Sherbrooke West, Montreal, QC, Canada H3A 2K6

E-mail address: clark@math.mcgill.ca 